\title{
New evidence of the Suess/de Vries cycle existing in historical naked-eye observations of sunspots
}

https://doi.org/10.1515/astro-2020-0004

Received Dec 05, 2019; accepted Mar 29, 2020

\begin{abstract}
Solar activity affects geophysical and heliophysical processes. Long-term changes in solar activity are closely related to climate change. Solar physicists and earth science researchers need longer observations of solar activity. Current continuous observations of solar activity are only about 400 years. Some scholars have compiled naked-eye observations of sunspots from $200 \mathrm{BC}$ to $1918 \mathrm{AD}$ from historical documents. In this present work, the authors use the weighted wavelet transform to study the observations series. The results show the Suess - de Vries cycle with a period from 195- to 235-year existing in the discontinuous sunspot series. Meanwhile, the cycle signal changes with time. Especially, the Suess/de Vries cycle is relatively obvious from $200 \mathrm{BC}$ to $400 \mathrm{AD}, 800 \mathrm{AD}$ to $1340 \mathrm{AD}, 1610 \mathrm{AD}$ to $1918 \mathrm{AD}$, with a period of about 211-year, about 195-year, and about 235-year, respectively.
\end{abstract}

Keywords: solar activity, Suess/de Vries cycle, weighted wavelet transform

\section{Introduction}

Solar activity depicts a dominant 11-year cyclic variability. Moreover, solar activity varies on other timescales, including short scale and long-term trends (Usoskin, 2017). These fluctuations affect the whole Earth system. At present, continuous observations of solar activity are available only for the last 400 years (Muñoz-Jaramillo \& Vaquero, 2019). To study the impact of long-term solar variations on the Earth system, solar activity observations covering longer time span are needed. Considering that the solar magnetic field shielded the Earth from the galactic particle showers, the stronger the cosmic rays, the higher the concentrations of the cosmogenic nuclides, which means the Sun is less active. Therefore, solar activity can be reconstructed based on cosmogenic nuclides including ${ }^{14} \mathrm{C},{ }^{10} \mathrm{Be}$ and ${ }^{36} \mathrm{Cl}$ (Usoskin et al., 2006; Usoskin, 2017). The cosmogenic proxy data is presently the only known way to get quantitative reconstructions of solar activity before 1610 . However, these data series are not direct solar observations, and its accuracy and reliability need to be continuously checked and verified.
Some scholars have found that naked-eye observations of sunspots are recorded in many historical documents. Based on this information, the sunspot number series can be obtained. It is a direct observation although it may be conditioned by meteorological and social factors. Moreover, the series is discontinuous. Based on this kind of historical observations, Vaquero et al. (2002) constructed naked-eye sunspot series from 200 BC to 1918 AD. They performed a spectral analysis of the series using the multitaper method (MTM) finding a strong peak at around 250 years that was confirmed using Singular Spectrum Analysis (SSA). Later, Ma \& Vaquero (2009) found a spectral peak of about 229 years in the yearly number of naked-eye observations of sunspots using a modified Lomb-Scargle periodogram analysis although its statistical significance was low. In this work, to further confirm the existence of the Suess/de Vries cycle, these naked-eye observations of sunspots are analyzed with weighted wavelet transform refining details of some periodic component. Moreover, the time variation of the Suess/de Vries cycle is also found in detail.

\section{Data}

Corresponding Author: Lihua Ma: National Astronomical Observatories, Chinese Academy of Sciences, Beijing 100101, China; Email: mlh@nao.cas.cn

José M. Vaquero: Departamento de Física, Universidad de Extremadura, Avda. Santa Teresa de Jornet 38, 06800 Mérida (Badajoz), Spain

○ Open Access. ( 2020 L. Ma and J. M. Vaquero, published by De Gruyter. Attribution 4.0 License
Naked-eye sunspot observations are the only direct information on historical activity from historical documents of the last two millennia (Arlt \& Vaquero, 2020). Fortunately, there are various catalogues of this kind of observations 
available in the scientific literature (Wittmann \& Xu, 1987; Yau \& Stephenson, 1988). Vaquero et al. (2002) use of these catalogues to construct a time series of the yearly number of observed sunspots (see, for example, the upper panel of Figure 1). Detailed explanations of the used criteria to generate the series are available in Vaquero et al. (2002). Note, in particular, that the observational dates were transformed to the Gregorian calendar. An important detail is that, for those periods of time with a lack of observations or records, the number of sunspots was originally evaluated equally to zero. However, we eliminate these invalid points from the yearly series and get uneven naked-eye observations of sunspots in this work. Note also that there is an information gap in the period 600-800 AD and a marked upward trend is detected in the series (see Ma \& Vaquero, 2009).

\section{Method}

The classic Fourier transform can give periodic components of a time series and display average effect of variability on different time scales during the whole period of time. In recent years, the wavelet transform uses expansion and translation techniques to gradually refine multi-scale change in the time series. It can automatically be adapted to requirements of time-frequency signal analysis and can focus on some details of the signal. With wavelet analysis, discrete signals with local characteristics can be approximated. The changes in the signal on a certain time scale can be reflected (Daubechies, 1992; Foufoula-Georgiou \& Kumar, 1994; Torrence \& Compo, 1998).

Actually, observations were often collected at uneven time. This resulted in uneven time interval existing in real observations. The series can be usually processed with the Lomb-Scargle periodogram. Based on principle of discrete Fourier transform, the Lomb-Scargle periodogram decomposes a time series into a series of linear combinations of sine (cosine) functions. Then the signal characteristics are transformed to frequency domain from time domain (Lomb, 1976; Scargle, 1982). This analysis can handle nonuniform sampling time series, but it only gives the overall effect. The conventional continuous wavelet transform can also analyze continuous observation series. In 1996, Foster proposed a weighted wavelet Z-transform (WWZ) that can directly detect period of non-uniform sampling time series (Foster, 1996a,b; An et al., 2016). Based on Morlet wavelet, the WWZ projects the signals onto three trial functions: a constant function $1(t)=1, \cos (\omega(t-\tau))$, and $\sin (\omega(t-\tau))$, where $\omega$ is the scale factor and $\tau$ is the time shift. The WWZ power is defined as $\frac{\left(N_{e f f}-3\right) V_{y}}{\left(2\left(V_{x}-V_{y}\right)\right)}$, where $N_{\text {eff }}$ is a quantity representing the local number density of the data, and $V_{x}$ and $V_{y}$ are the weighted variations of the data and the model functions, respectively. For more details on the methodology, see Foster (1996b). The WWZ transform can investigate discontinuous series and reveal change of signals on a certain time scale through localization analysis of time and frequency. Here it is used to analyze the periodicity of the historical naked-eye observations of sunspots. Meanwhile, results of the Lomb-Scargle periodogram are also achieved in the next section.

\section{Wavelet Analysis}

Considering that those periods of time with a lack of observations or records, the sunspots were evaluated with zero value in the time-series of naked-eye observations. Firstly, the actual observations of naked-eye sunspots can be obtained after excluding time points with zero value in the series. The original naked-eye observations of sunspots from $200 \mathrm{BC}$ to $1918 \mathrm{AD}$ are plotted on the top of Figure 1. The subfigures in the bottom of Figure 1 give the LombScargle periodogram (left) and WWZ spectrum (right), respectively. In Figure 1, the sunspot observations and the WWZ spectrum are sharing the same abscissa time scale, and Lomb-Scargle periodogram and WWZ spectrum are sharing the same ordinate frequency scale.

In the results of the WWZ spectrum, the color scale represents the Z-statistics of the WWZ power of a certain period at a given time. It is corresponding to the intensity of the power spectrum. Warm red and cold blue colors are indicating strong signals and weak signals, respectively. It can be seen from the WWZ spectrum in Figure 1 that the Suess - de Vries cycle is a significant signal in the nakedeye sunspot series. Meanwhile, periodic characteristics of the signal change significantly over time. Especially, the signal is the strongest from $800 \mathrm{AD}$ to $1340 \mathrm{AD}$, and the peak power appears in about $1060 \mathrm{AD}$ with a period length of 195 years. From 200 BC to 400 AD, from 1610 AD to 1918 AD, the Suess de Vries cycle is also obvious, and the length of the cycle corresponds to 211 years and 235 years, respectively. In other periods of time, the signal is very weak in the WWZ spectrum. The Lombs-Scargle periodogram also detects the Suess/de Vries cycle with an average period length of about 231 years. This is generally consistent with the results of wavelet analysis.

Considering a time gap from $600 \mathrm{AD}$ to $800 \mathrm{AD}$ existing in the naked-eye sunspots series (Stephenson, 1990), here the series from $800 \mathrm{AD}$ to $1918 \mathrm{AD}$ is analyzed. The results 


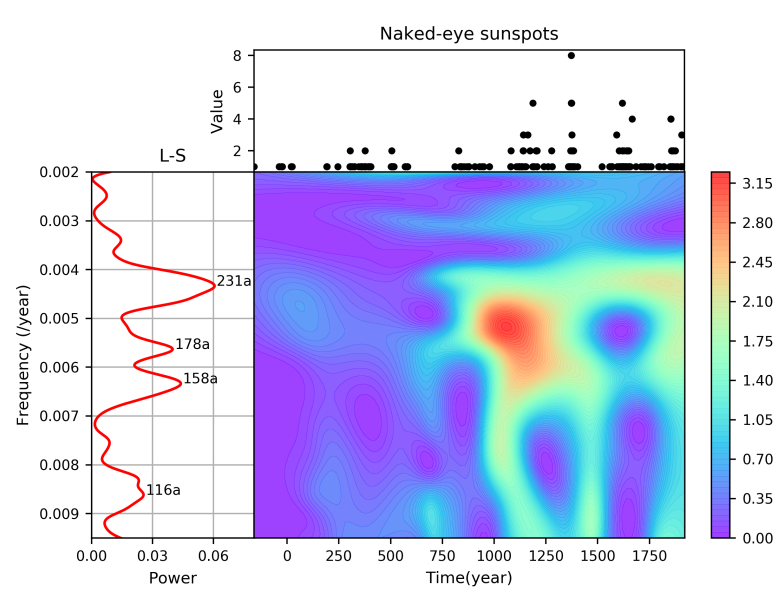

Figure 1. The weighted wavelet Z-transform (WWZ) results and the Lomb-Scargle periodogram of the naked-eye observations of sunspots from $200 \mathrm{BC}$ to $1918 \mathrm{AD}$.

are shown in Figure 2. The meaning of each subfigure is the same as that of Figure 1.

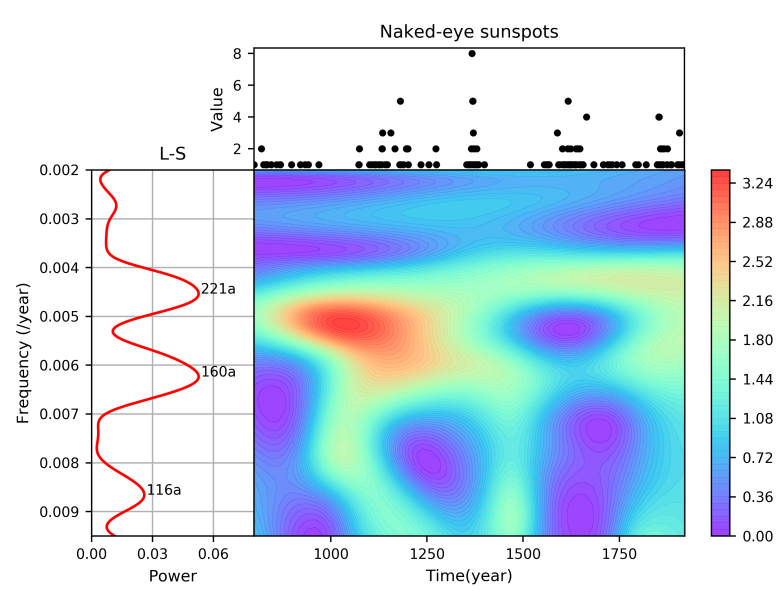

Figure 2. The weighted wavelet Z-transform (WWZ) results and the Lomb-Scargle periodogram of the naked-eye observations of sunspots from 800 AD to 1918 AD.

In Figure 2, the remarkable Suess - de Vries cycle appears from $800 \mathrm{AD}$ to $1340 \mathrm{AD}$ and $1610 \mathrm{AD}$ to $1918 \mathrm{AD}$, respectively. The periodic length is corresponding to 195- and 235-year, respectively. The signal is significantly stronger from $800 \mathrm{AD}$ to $1340 \mathrm{AD}$ than during $1610 \mathrm{AD}$ to $1918 \mathrm{AD}$. This is very consistent with the analysis results of the series from $200 \mathrm{BC}$ to $1918 \mathrm{AD}$. Even with the short series, the analysis results are not affected.

In the Lomb-Scargle periodogram of Figure 2, it can be seen that the length of the Suess - de Vries cycle becomes 221 years. This is different from the results (231 years) obtained from data series during $200 \mathrm{BC}$ to $1918 \mathrm{AD}$. It can be confirmed that the Lomb-Scargle analysis results are highly related to the time span of the data series used, especially for observations with obvious time variability. Wavelet transform is a better choice of the data series analysis.

\section{Conclusion and Discussion}

Based on results of the weighted wavelet Z-transform (WWZ) of the naked-eye observations of sunspots, it is further confirmed that the Suess/de Vries cycle is detected in solar activity. Meanwhile, the fluctuation has significant timevarying characteristics. The periodic signal apparently appeared in $200 \mathrm{BC}$ to $400 \mathrm{AD}, 800 \mathrm{AD}$ to $1340 \mathrm{AD}, 1610 \mathrm{AD}$ to $1918 \mathrm{AD}$, respectively. The corresponding periods of the signal are 211, 195, and 235 years, respectively. The signal is the most prominent during $800 \mathrm{AD}$ to $1340 \mathrm{AD}$, and the power peak appears in about $1060 \mathrm{AD}$.

With Lomb-Scargle periodogram analysis, Ma \& Vaquero (2009) investigated the periodicities in naked-eye observations of sunspots during 200 BC - 1918 AD. Results confirmed that the Suess/de Vries cycle with about 229 years exists in solar variability. Actually, analysis results with the Lomb-Scargle periodogram are related to the frequency intervals adopted to a large extent. It is possible that there is several-year error during analysis of the Suess/de Vries cycle length. In order to display spectrum in detail, we analyze more frequency intervals in this work. Therefore, the Lomb-Scargle periodogram is quite different from the previous study (Ma \& Vaquero, 2009). The next fundamental step is to establish the statistical significance of these results obtained with the WWZ transform that cannot currently be estimated.

Acknowledgment: The authors are grateful to M. Emre Aydin for providing a weighted wavelet Z-transformation analysis program. This work was partly funded by FEDER-Junta de Extremadura (research group grant GR18097 and project IB16127) and from the Ministerio de Economía y Competitividad of the Spanish Government (CGL2017-87917-P).

\section{References}

An T, Lu XL, Wang JY. 2016. Temporal evolution of long-timescale periodicities in ULX NGC 5408 X-1. Astron Astrophys 585:A89. Arlt R, Vaquero JM. 2020. Historical sunspot records. Living Rev Sol Phys 17(1):1. 
Daubechies I. Ten Lectures on Wavelets. Philadelphia: SIAM; 1992. https://doi.org/10.1137/1.9781611970104.

Foster G. 1996a. Time series analysis by projection. I. statistical properties of Fourier analysis. Astron J. 111:541.

Foster G. 1996b. Wavelets for period analysis of unevenly sampled time series. Astron J. 112(4):1709 https://doi.org/10.1086/118137.

Foufoula-Georgiou E, Kumar P. Wavelets in Geophysics. San Diego: Academic Press; 1994.

Lomb NR. Least-squares frequency analysis of unequally spaced data. Astrophys Space Sci. 1976;39(2):447-462.

Ma LH, Vaquero JM. 2009. Is the Suess cycle present in historical naked-eye observations of sunspots? New Astron. 14(3):307 https://doi.org/10.1016/j.newast.2008.09.003.

Muñoz-Jaramillo A, Vaquero JM. 2019. Visualization of the challenges and limitations of the long-term sunspot number record. Nature Astronomy 3(3):205-211.

Scargle JD. 1982. Studies in astronomical time series analysis. II - Statistical aspects of spectral analysis of unevenly spaced data. Astrophys J. 263:835-853.
Stephenson FR. 1990. Historical evidence concerning the Sun: interpretation of sunspot records during the telescopic and pretelescopic eras. Phil. Trans. R. Soc. Lond. A. 330(1615):499512.

Torrence C, Compo GP. 1998. A practical guide to wavelet analysis. Bull Am Meteorol Soc. 79(1):61-78.

Usoskin IG. 2017. A history of solar activity over millennia. Living Rev Sol Phys. 14(1):3.

Usoskin IG, Solanki SK, Korte M. 2006. Solar activity reconstructed over the last 7000 years: the influence of geomagnetic field changes. Geophys Res Lett. 33(8):L08103.

Vaquero JM, Gallego MC, Garcia JA. 2002. A 250-year cycle in nakedeye observations of sunspots. Geophys. Res. Lett. 29(20):58.

Wittmann AD, Xu ZT. 1987. A catalogue of sunspot observations from 165 BC to AD 1684. Astron Astrophys Suppl Ser. 70:83-94.

Yau KKC, Stephenson FR. 1988. A Revised Catalogue of Far Eastern Observations of Sunspots (165 BC to AD 1918). Q. J. R. Astr. Soc. 29:175. 